\title{
Publishing landscape ecology research in the 21st century
}

\author{
Eric J. Gustafson
}

Received: 11 March 2011 / Accepted: 13 July 2011 / Published online: 2 August 2011

(C) Springer Science+Business Media B.V. (outside the USA) 2011

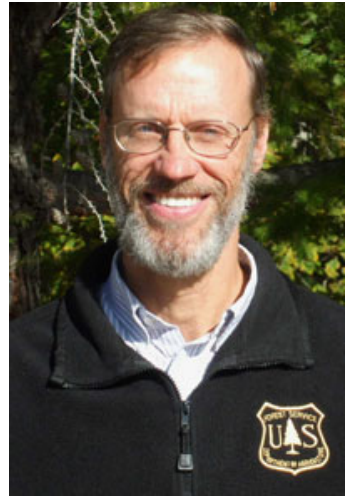

\section{The problem}

With the proliferation of journals and scientific papers, it has become impossible to sustain a familiarity with the corpus of ecological literature, which totals tens of thousands of pages per year. Given the number of papers that a well-read ecologist should read, it takes an inordinate amount of time to extract the critical details necessary to superficially understand and evaluate the significance of a study from the typical journal article. Determining if a

\section{E. J. Gustafson ( $\square)$}

Institute for Applied Ecosystem Studies, USDA Forest

Service, Northern Research Station, Rhinelander,

WI, USA

e-mail: egustafson@fs.fed.us paper is worth taking the time to read closely is becoming more important as the number of published papers increases. Now that the transition to the digital age is nearly complete, perhaps the time is ripe for this situation to be changed.

The traditional format for an ecological journal paper reflects the scientific and publishing technology of 50 years ago. The traditional format for publishing a scientific study assumes that studies are fairly simple and that the paper will contain sufficient detail for an independent researcher to reproduce the study. For more complex studies, such papers might include an appendix with necessary ancillary information. However, a majority of landscape ecological studies today include some combination of complex data collection campaigns, massive electronic datasets, sophisticated spatial data processing procedures, complex computer models, highly technical (and sometimes novel) analytical techniques, a major extension of an established technique or model, multiple large study areas, novel combinations of existing approaches, and in some cases, all of the above! This situation obviously stretches the capabilities of the traditional publication format. In fact, because of journal length limits, most papers fail to provide sufficient information to allow an independent researcher to reproduce the study, even with an appendix or two.

In my experience as an editor and reviewer, a surprisingly high number of submissions to ecological journals do not present a rigorous scientific 
analysis that produces conclusive results. Excluding methods development papers, these are either descriptive studies, case studies presented without conclusive analysis, or they fail to definitively test a meaningful hypothesis. Speculation is also common, and in many cases it masquerades as conclusion. When I am evaluating a study, I often become frustrated that I can not readily find the answers to the critical questions about a study in the typical journal article. Questions such as, what is the specific scientific question being addressed? What hypotheses are being tested? What are the assumptions made in the experimental design and analysis? How were data used to confront the hypotheses? How did the hypotheses fare? What conclusions were drawn and do they follow from the data and analysis? What uncertainties remain? Sometimes the answers are buried in the text, but often they are not clearly identified or articulated. One reason for this is that the editorial practices of ecological journals do not compel authors to clearly state this information. We specifically ask for only five vaguely defined sections: an introduction, a description of the methods, the results, a discussion and a list of references. Many journals list other requirements in their guidelines for authors, but there is no formal mechanism to ensure that they appear in the published paper. I believe we could do a better job of ensuring that the fundamental details of a study are present and easily located in published papers.

\section{Some ideas for a solution}

How can we improve this situation? One option would be to add specific items to the list of required sections, perhaps allowing authors some flexibility in the order in which they appear in a manuscript. Another option would be to require authors to provide text for a sidebar or box containing fundamental information about the study. This would be analogous to the "In a Nutshell" box that is part of every paper in the Ecological Society of America journal Frontiers in Ecology and the Environment, although I am envisioning something much more substantive and useful than what appears there. A related option would be to require that each of the critical questions listed above be answered in the abstract.
There is also an opportunity to greatly improve the usefulness of published work by harnessing electronic and on-line technology to revise the publication format. Most journals already publish on-line supplemental material to accompany a paper, which provides many of the details needed to reproduce a study. Most journals also concurrently publish papers on-line, and many of these contain hyperlinks to other parts of the paper, or to related material (e.g., cited papers). I propose that we further redesign the publication format for ecological research that (1) makes it easier for readers to find the fundamental details of a study, (2) shows readers only the details in which they are interested, (3) allows for publication of unlimited detail and ancillary material, (4) is more accessible to researchers worldwide and (5) phases out the reliance on printed material.

What would a re-designed publication format look like? I do not presume to have a definitive answer, but I do have some ideas that will hopefully start a conversation that might lead to something better than the status quo. I see two primary areas of focus. (1) Revise the list of required items that must be included in a manuscript submitted for publication and (2) develop a new format for publishing papers that is amenable to both paper and electronic media. The former would improve the quality of communication of scientific research to other researchers and practitioners. The latter would make the dissemination of scientific knowledge less costly, more time efficient and more accessible to researchers, practitioners and policy makers.

The traditional format for presenting scientific research consists of four major sections: Introduction, Methods, Results and Discussion. Considerable discretion is given to authors about what to include in these sections, and there is currently no mechanism to compel authors to clearly state the fundamental characteristics of their study. Here I propose a somewhat expanded format (seven major sections) that attempts to resolve some of these problems by explicitly forcing authors to communicate the fundamental characteristics of their study, and separates these from the more technical details.

1. Statement of problem

a. What is the problem being addressed in this paper? 

b. What is the specific scientific question being addressed?
c. What is currently known about this question?
d. What do we need to know about this question and why?

2. Analytical framework (how the study produces conclusive results)
a. Description of the experimental design/meta- analysis/model development and testing/case study/method development
b. Listing of hypotheses tested, or some state- ment about how the study will formally and conclusively advance knowledge
c. List of assumptions
d. Analytical techniques that were applied
e. A priori criteria for drawing conclusions

3. Confronting the hypotheses with data (or formally advancing knowledge)
a. Study area
b. Data collection/existing data set (source, characteristics)
c. Analysis of appropriateness of data for analytical techniques (do they meet analyt- ical assumptions?)
d. Specifics about implementing the analytical procedures

4. Results
a. Outcome of applying the a priori criteria for drawing conclusions
b. Any heuristic results

5. Caveats

a. What are the potential implications of the assumptions used?

b. Was the analytical framework indeed appropriate?

c. What are the remaining uncertainties?

d. What is the scope of inference and application? Geographic? Taxa?

6. Discussion
a. What do the results mean?
b. How do these results compare to those reported from other studies?
c. How might the results be applied?
d. What are the next research questions?

\section{Formal conclusions}

a. What specific advances in knowledge were made (i.e., what was learned)?

b. What cannot be concluded from the data and analysis?

Perhaps this format should not be prescriptive, but should define the editor's expectations for authors. For example, the sections and sub-sections could appear in any logical order, allowing the format to be applicable to a wide variety of studies. Perhaps the outline above could form a template for the paper submission cover letter, providing editors and reviewers with a comprehensive, yet concise summary of the fundamental details of the study.

One drawback of the traditional printed format for journal articles is that it is inherently linear, and readers must wade through details they may not presently be interested in, often missing information that they are interested in. Print journals also require the imposition of sometimes restrictive length limits. In the 21 st century, we should take full advantage of electronic technology, and the printed version should be an abridged version of the electronic version with a URL address to access the full version. Therefore, electronic versions of a paper should feature collapsible/expandable sections and extensive use of hypertext. Perhaps the URL would open with all sections collapsed except the abstract and the sidebar containing the fundamental study details. The reader can then expand the sections they are interested in, in the order in which they become interested in them. Similarly, hyperlinks can provide easy access to other parts of the paper (including lengthy appendix and archive information) and to cited works. Eventually, links to specific sections or even sentences of other papers could be inserted into the text. This would make the reading of a paper less inherently linear, allowing readers to access information in whatever order suits their purpose. It would also allow readers to more readily find the material that is of interest, to bypass material that is not of interest, reducing the time needed to extract the information they seek.

I have argued that many readers would value a succinct summary of the fundamental characteristics of a study. Such a summary could be required of the authors and placed in a sidebar or text box in a prominent place in the article. It might best take the form of a bulleted list. Hyperlinks to the relevant text 
within the article would make this an effective way to access the relevant content of the paper. An alternative would be to have links from a generic, standard list of the fundamental characteristic of research studies to the places in the text that describe those characteristics. This list would function like a hyperlinked table of contents.

Papers become quite lengthy when they provide all the information necessary for another researcher to repeat the study, and journal length limits sometimes force omission of some information. Furthermore, inclusion of details impedes the prose conducive to communicating the primary components of most studies. An electronic publication format can theoretically allow publication of unlimited amounts of detail with non-sequential access via hyperlinks. This detail could include a repository of files that would allow replication of the study. For modeling studies, this might include parameter and input files, and even executable files. For field studies it might include detailed data collection protocols and ancillary data sets (with metadata).

I predict that the printed versions of scientific journals will eventually be phased out entirely. But, assuming that printed versions of papers will persist for some time as we transition to all-electronic publication, I propose that print versions of papers be abridged, perhaps to a maximum length of five pages. Perhaps the easiest way to accomplish this is to print a collapsed version of the paper. Authors would compose each section knowing that only the first paragraph would appear in the printed (and collapsed, on-line) version. The printed version would also include the URL to the complete on-line version.

\section{Potential outcomes}

If such a vision were implemented, I see a number of positive outcomes for landscape ecologists. (1) We would be able to read and assimilate more of the ecological literature, (2) the communication of research results would be more effective, (3) the quality of research may improve as authors are compelled to more explicitly describe the scientific rigor of their studies, (4) research results will be more accessible throughout the world, (5) there will be a reduction in the cost and time for publishing scientific work, (6) practitioners will be better able to access scientific information from the literature and (7) more studies will be replicated and more meta-analyses conducted because the information necessary to do so will be included in all papers.

The future of scientific publishing and dissemination is being vigorously discussed in virtually every field of scientific endeavor. However, there is no need for landscape ecologists to wait until scientists in other disciplines blaze a trail. Our field is small enough that we can develop, test and implement a solution that advances landscape ecology. The journal Landscape Ecology is certainly well-positioned to lead by example. My goal is to stimulate a discussion about options and see if a consensus emerges. Perhaps the editorial pages of this journal can serve as a forum for such a dialogue. Let the discussion begin!

Acknowledgments I thank Monica Turner and Geoff Henebry for their insightful comments. 